Research Article

\title{
On the Capacity of Densely Packed Arrays with Mutual Coupling and Correlated Noise
}

\author{
Vahid Dehghanian and John Nielsen \\ Electrical and Computer Engineering Department, University of Calgary, Calgary, AB, Canada T2N 1N4 \\ Correspondence should be addressed to Vahid Dehghanian; vdehghan@ucalgary.ca
}

Received 30 March 2015; Accepted 17 August 2015

Academic Editor: Ahmad Safaai-Jazi

Copyright ( 2015 V. Dehghanian and J. Nielsen. This is an open access article distributed under the Creative Commons Attribution License, which permits unrestricted use, distribution, and reproduction in any medium, provided the original work is properly cited.

\begin{abstract}
Capacity of a wireless link can be enhanced by increasing the number of receive antennas. However, imposed receiver physical size constraints necessitate that the antenna elements be in close proximity, which typically reduces the overall link capacity of the wireless channel. Counterintuitively, under certain conditions the capacity of the overall link can be enhanced by decreasing antenna spacings. The focus of this paper is that of identifying the fundamental mechanisms and the conditions that give rise to this excess capacity. Closed-form expressions that directly quantify this capacity gain are derived based on a representative circuit theoretic model. Interesting insights are developed about the impact of different noise and interference sources and the limiting effect of heat losses in the antenna system. The capacity analysis is subsequently generalized to encompass the effect of antenna current deformation and load mismatch due to mutual coupling, based on the standard Method of Moments (MoM) analysis, demonstrating similar capacity enhancement behavior as predicted by the closed-form expressions.
\end{abstract}

\section{Introduction}

The maximum rate of information transfer through a communications link is quantified by the mutual information shared between the transmitter and the receiver. The mutual information can be optimized by matching the transmitter source coding and modulation to the channel, which attains the channel capacity [1, pp. 183-241]. In this paper, references made to capacity presuppose such channel matching. It is well known that the capacity of a wireless channel can be significantly enhanced by the use of multiple element arrays (MEAs) in the form of single input multiple output (SIMO) or multiple input multiple output (MIMO) systems [2]. MEAs have been extensively investigated, implemented, and published over the past two decades and it is beyond the scope of this paper to provide an overview of this extensive area of research. A general observation is that while the channel capacity increases approximately linearly with the number of antenna elements, it is ultimately limited by the overall physical size of the MEA [3-8]. This is a fundamental limitation of MEAs incorporated into mobile handheld receivers in which the physical size constraints result in small antenna spacings.
Most prior studies on closely spaced antenna systems (e.g., [3] and the references therein) focus on the signal spatial correlation (SSC), which increases with smaller interelement spacings. The terms "closely spaced" and "densely packed" in this paper are interchangeably used and will be taken to mean that the MEA elements are spaced less than half a carrier wavelength apart.

More recently, the issue of mutual coupling, which occurs due to electromagnetic interaction between MEA elements, has received more attention [4-8]. In most of these publications mutual coupling has been typified as a deteriorating factor that results in increased SSC [4-6] and bigger return losses $[7,8]$, hence a reduced capacity. But counter to the generally accepted notion that increased mutual coupling results in capacity loss, $[9,10]$ have reported increased capacity in the MIMO system comprising closely spaced coupled antennas relative to the case involving widely separated noninterfering elements. Reference [9] has ascribed this capacity gain to the decreased SSC at certain nonzero values of mutual coupling. This argument, however, is contrary to other related works, for example, $[4,5]$, that have reported monotonically increasing SSC with increasing mutual coupling. 
Reference [10] has attributed this capacity gain to the increased effective aperture of the antenna system at small spacings based on full-wave electromagnetic analysis, which, due to the numerical analysis details, partially obfuscates the underlying principles. Our in-depth analysis of channel capacity is aimed at clarifying these conflicting reports and is a contribution of this paper. As aptly shown by Cover and Thomas [1, pp. 183-241], the capacity of a MIMO communications channel cannot be increased by a weighted combining of the individual conducted channels. Furthermore, a combining process that is not isomorphic, such that it is not invertible, generally results in a loss in mutual information that deviates further from the attainable capacity. However, while this is applicable to conducted MIMO signals it is not directly applicable to the combining via mutual coupling of antenna elements. The transition from radiated to conducted energy via an antenna element is a lossy process in that the radiation mode of the receiving antenna is invariably significantly mismatched to the mode of the incident electromagnetic field. This modal mismatch loss is serendipitously partially ameliorated by the mutual coupling that invariantly exists between antenna elements of close proximity, resulting in the observed capacity gain. This concept will be exemplified analytically in Section 3 of this paper based on an idealized SIMO system consisting of identical electrically short dipole antennas at the receiver. The approximate simplified expressions developed in Section 3 of this paper illuminate the underlying mechanisms of capacity enhancement in densely packed arrays and are a contribution of this paper. The SIMO system allows us to exploit the linearity of the receiver processing and develop several insightful closed-form expressions. These expressions illuminate the underlying mechanisms of the capacity enhancement achievable with the multiple element closely spaced antenna systems (MECSA) relative to the case with zero mutual coupling, that is, the widely separated multiple element noninterfering antenna system (MENIA), and are the main contribution of this paper. Had we considered MIMO systems for our analysis, channel capacity relations would have involved nonlinear processing (e.g., water-filling) that would obscure the essence of this capacity gain.

The increase in MEA's effective aperture at small interelement spacings has been known to the antennas and propagation community as array superdirectivity for many years but its implication on channel capacity has only been considered relatively recently. As shown in [11] the optimum space time (ST) processing that achieves the capacity of a SIMO channel, under Gaussian channel noise and Gaussian source entropy, is equivalent to the processing that results in maximum MEA directivity. As such, the ST processing that achieves the capacity of the communications channel may derive the densely packed array into a superdirectivity regime. The problem is, however, that superdirectivity is associated with low efficiency, for example, narrow bandwidth and high sensitivity to beamforming weights. References [12, 13] have developed a frame work to compute capacity while constraining the tolerable level of superdirectivity. While these publications and the references therein have reported important finding about array superdirectivity and its impact on channel capacity, nonetheless, these studies have not incorporated detailed models of receiver front end and noise in their studies. Fundamental to the capacity analysis is the statistics of the channel noise, which ultimately sets the signal to noise ratio achievable by the MEA and hence the capacity. In this paper, we adopt a network theory framework similar to [14] to model the noise generated internally within the receiver, which will be described in Section 2. We note that [15] has also studied the impact of mutual coupling on channel noise and its implication on MIMO system capacity; however, the results therein take a different approach based on establishing front-end design principles for MIMO receivers.

How channel capacity is influenced by mutual coupling under dominating receiver amplifier noise and spatially white interference has been discussed in [11] and [16], respectively. But heat losses in the antenna system, which as will be shown in Section 3 of this paper significantly impact the achievable capacity of densely packed antenna systems, have not been incorporated into the developed capacity relations in these publications. We note that the authors in [13] have incorporated antenna heat losses in their analysis of channel capacity. But the capacity formulations developed in [13] ignore the impact of receiver circuit and noise correlation; hence, the achievable capacity of a densely packed MEA system is not clear from the results developed therein. Also, to the best of authors' knowledge, the question of highly directional interference (HDI) and its impact on the capacity of closely spaced antenna systems has not been treated before. Our theoretical analysis of channel capacity involves different scenarios of channel noise including the dominant HDI case. The "HDI" in this paper refers to interfering signals that arrive from a narrow spatial angle, for example, interference due to a nearby transmitter or a jammer.

The impact of element spacing on the usable bandwidth of densely packed arrays has been extensively studied in [12] and the references therein. But, for the sake of completeness, the impact of element spacing on the array bandwidth of operation will be briefly discussed in Section 3 . In Section 4, extensions will be made to analysis of practical implementable antenna elements based on the standard MoM. The MoM analysis illustrates the impact of antenna current deformation on channel capacity, which is another contribution of this paper. The behavior of the capacity, observed based on the numerical MoM analysis, closely resembles the predictions made by the developed closedform relations. Hence the understanding of the fundamental mechanisms of the capacity enhancement pervades into more generalized configurations. The impact of load mismatch on the achievable capacity of MECSA systems is also discussed in Section 4. Conclusions are provided in Section 5.

\section{System Model}

A SIMO system is considered, which consists of a single transmit antenna that is located far from an $M$-element receive MEA such that the transmit antenna pattern is not influenced by loading and mutual coupling in the receive MEA and vice versa. The transmit and the receive antennas are taken to be linearly polarized antennas aligned with 
the propagated electric field which travels through a linear, passive, and isotropic medium, in the horizontal plane. The receive MEA elements are taken to be identical electrically short dipoles made from perfectly conducting thin wires. Electrically short implies that the antenna scatters negligibly when open-circuited and that the current distribution on its conductor is not affected by nearby scattering objects, mutual coupling to other antennas, and loading.

The source entropy of the transmitter is represented by a carrier modulated pulse function that is modulated by the random data-dependent complex symbol, denoted by $s$, which has a zero-mean Circularly Normal (CN) density, also known as circularly symmetric complex normal density. A power constraint is imposed such that the radiated power density in the direction of the receive MEA is $\mathrm{E}_{s}\left[s s^{*}\right]=\mathcal{E}$, where $\mathrm{E}_{x}[]$ denotes the expectation with respect to " $x$ " and the superscript “*” denotes the complex conjugate.

The transmit signal is assumed to be narrowband such that the antenna impedances are approximately constant (not frequency-dependent) over the extent of the signal bandwidth. Narrowband also subsumes that the maximum propagation delay across the physical dimensions of the receive MEA is much smaller than the inverse of the signal symbol bandwidth. As such the ST processing can be done separately in the temporal and the spatial domains [17, pp. 352-64]. To focus the analysis, henceforth the temporal processing (e.g., signal demodulation) is implied.

The complex vector of baseband voltages across opencircuit antenna terminals is given as

$$
\mathbf{v}_{\mathrm{o}}=s \mathbf{h}_{\mathrm{S}}+\mathbf{v}_{\mathrm{EN}}
$$

where $\mathbf{h}_{\mathrm{S}} \in \mathbb{C}^{M}$ is the $M \times 1$ vector of instantaneous complex channel gains which incorporates all of the spatial characteristics of the incident electromagnetic wave. Under free space conditions $\mathbf{h}_{\mathrm{S}}$ embodies the signal direction induced on the receive MEA by the incoming signal plane wave. Under scattering conditions with large angular spread at the receiver, $\mathbf{h}_{\mathrm{S}}$ will have a zero-mean jointly $\mathrm{CN}$ density. A normalization of channel gains is considered such that $\mathbf{h}_{\mathrm{S}}^{H} \mathbf{h}_{\mathrm{S}}=M$ for free space and $\mathrm{E}_{\mathbf{h}_{\mathrm{S}}}\left[\mathbf{h}_{\mathrm{S}}^{H} \mathbf{h}_{\mathrm{S}}\right]=M$ for scattering, which maps the transmit power constraint into the intensity of the incident electromagnetic signal at the receiver. The $M \times 1$ vector $\mathbf{v}_{\mathrm{EN}} \in$ $\mathbb{C}^{M}$ represents the vector of open-circuit antenna voltages due to cochannel interfering signals that are intercepted by the receiver antennas. These radiated interference signals are denoted in this paper as extrinsic noise (EN). In general, EN may include thermal radiation, cosmic background (sky noise), and cochannel HDIs due to other transmitters and electronic devices in the channel.

The transfer of signal and noise power between the MEA and the $M$ uncoupled identical receiver channels is controlled using a lossless multiport decoupling network, also known as multiport matching network [18], as shown in Figure 1. The impact of load mismatch will be treated in Section 4. Although lossless multiport matching networks are practically difficult to implement, they provide a convenient theoretical construct that allows us to study the impact of small interelement spacing on the signal power collection capability and noise characteristics of MECSA. In practice, suboptimal matching strategies, such as the adaptive uncoupled matching presented in [19], may be used to mitigate the negative impact of impedance mismatch on the data throughput of the communications link. As will be demonstrated in Section 4, the capacity of a SIMO system can still be enhanced with mutual coupling even if no optimization of the multiport impedance network is assumed. However, this is not necessarily true for the MIMO case where accurate multiport matching is crucial to realize enhanced gain with mutual coupling. In a MIMO system, multiport matching not only eliminates the losses due to impedance mismatch but also decouples the antennas, hence, making spatial multiplexing possible even as antenna spacing approaches zero [20]. A possible lossless multiport conjugate impedance match is one that results in the maximum signal energy transfer to receiver output loads, denoted by $z_{\mathrm{L}}$. An ideal invertible matching network may be given as [14]

$$
\left[\begin{array}{ll}
\mathbf{Z}_{M 11} & \mathbf{Z}_{M 12} \\
\mathbf{Z}_{M 21} & \mathbf{Z}_{M 22}
\end{array}\right]=j\left[\begin{array}{cc}
-\mathbf{X}_{\mathrm{A}} & \left(r_{0} \mathbf{R}_{\mathrm{A}}\right)^{1 / 2} \\
\left(r_{0} \mathbf{R}_{\mathrm{A}}\right)^{1 / 2} & x_{0} \mathbf{I}
\end{array}\right] \text {, }
$$

where $\mathbf{I} \in \mathbb{R}^{M \times M}$ denotes the $M \times M$ identity matrix, $\mathbf{R}_{\mathrm{A}}, \mathbf{X}_{\mathrm{A}} \in \mathbb{R}^{M \times M}$ denote the resistance and reactance matrices of the MEA (i.e., $\mathbf{Z}_{\mathrm{A}}=\mathbf{R}_{\mathrm{A}}+j \mathbf{X}_{\mathrm{A}}$ ), and $z_{0}=r_{0}+j x_{0}$ is the impedance looking into port $m \in\{1, \ldots, M\}$ of the matching network from the receiver side and may be tuned to minimize the receiver noise figure $[14,20]$. Also, $\mathbf{A}^{1 / 2}=$ $\mathbf{V}(\mathbf{A}) \boldsymbol{\Lambda}(\mathbf{A})^{1 / 2} \mathbf{V}(\mathbf{A})^{H}$ denotes the positive definite square root of the positive definite matrix $\mathbf{A} . \mathbf{V}(\mathbf{A})$ is the matrix of eigenvectors, $\boldsymbol{\Lambda}(\mathbf{A})$ is the diagonal matrix of eigenvalues of $\mathbf{A}$, and superscript " $H$ " denotes the Hermitian transpose. From reciprocity and conservation of energy, $\mathbf{R}_{\mathrm{A}}$ is symmetric and has positive real eigenvalues and, hence, is positive definite. Since $\mathbf{R}_{\mathrm{A}}$ is positive definite, it is invertible and its inverse, $\mathbf{R}_{\mathrm{A}}^{-1}$, is also positive definite. The antenna array plus the matching network can then be represented by a Thevenin equivalent circuit with $\mathbf{v}_{\mathrm{Th}}=\sqrt{r_{0}} \mathbf{R}_{\mathrm{A}}^{-1 / 2} \mathbf{v}_{\mathrm{o}}$ and $\mathbf{Z}_{\mathrm{Th}}=z_{0} \mathbf{I}[21]$.

We assume that there is no coupling between channel electronics such as amplifiers and mixers. That is, coupling only occurs between the antennas. This ignores the possibility of receiver channel noise correlation due to phase noise of common oscillator signals used for downconversion and sampling. With this approximation, the receiver channels downstream the multiport matching network are modeled by a bank of identical and uncoupled linear noisy two-port networks based on the Rothe-Dahlke model [22], as shown in Figure 1. Accordingly, noise generated internally within each receiver channel is represented by independent voltage and current sources, $v_{\mathrm{a} m}$ and $i_{\mathrm{a} m}$, which have zero-mean $\mathrm{CN}$ densities. That is, $\mathrm{E}_{\mathbf{v}_{\mathrm{a}}, \mathbf{i}_{\mathrm{a}}}\left[\mathbf{v}_{\mathrm{a}} \mathbf{i}_{\mathrm{a}}^{H}\right]=\mathbf{0} \in \mathbb{R}^{M \times M}$ and $\mathrm{E}_{\mathbf{v}_{\mathrm{a}}}\left[\mathbf{v}_{\mathrm{a}} \mathbf{v}_{\mathrm{a}}^{H}\right]=$ $\sigma_{v}^{2} \mathbf{I}, \mathbf{E}_{\mathbf{i}_{\mathrm{a}}}\left[\mathbf{i}_{\mathrm{a}} \mathbf{i}_{\mathrm{a}}^{H}\right]=\sigma_{i}^{2} \mathbf{I}$, where $\left[\mathbf{v}_{\mathrm{a}}\right]_{m}=v_{\mathrm{a} m}$ and $\left[\mathbf{i}_{\mathrm{a}}\right]_{m}=i_{\mathrm{a} m}$.

The circuit in Figure 1 may be solved to obtain the voltages across output loads $z_{\mathrm{L}}$, as $\mathbf{v}_{z_{\mathrm{L}}}=\mathbf{G}\left(\mathbf{v}_{\mathrm{Th}}-\mathbf{v}_{\mathrm{IN}}\right)$, where $\mathbf{v}_{\mathrm{IN}}=$ $\mathbf{v}_{\mathrm{a}}+\left(z_{0}+z_{\text {cor }}\right) \mathbf{i}_{\mathrm{a}}$ represents the $M \times 1$ vector of intrinsic noise (IN) voltages due to noisy electronics downstream the matching network. The impedance $z_{\text {cor }}$ is the Rothe-Dahlke 


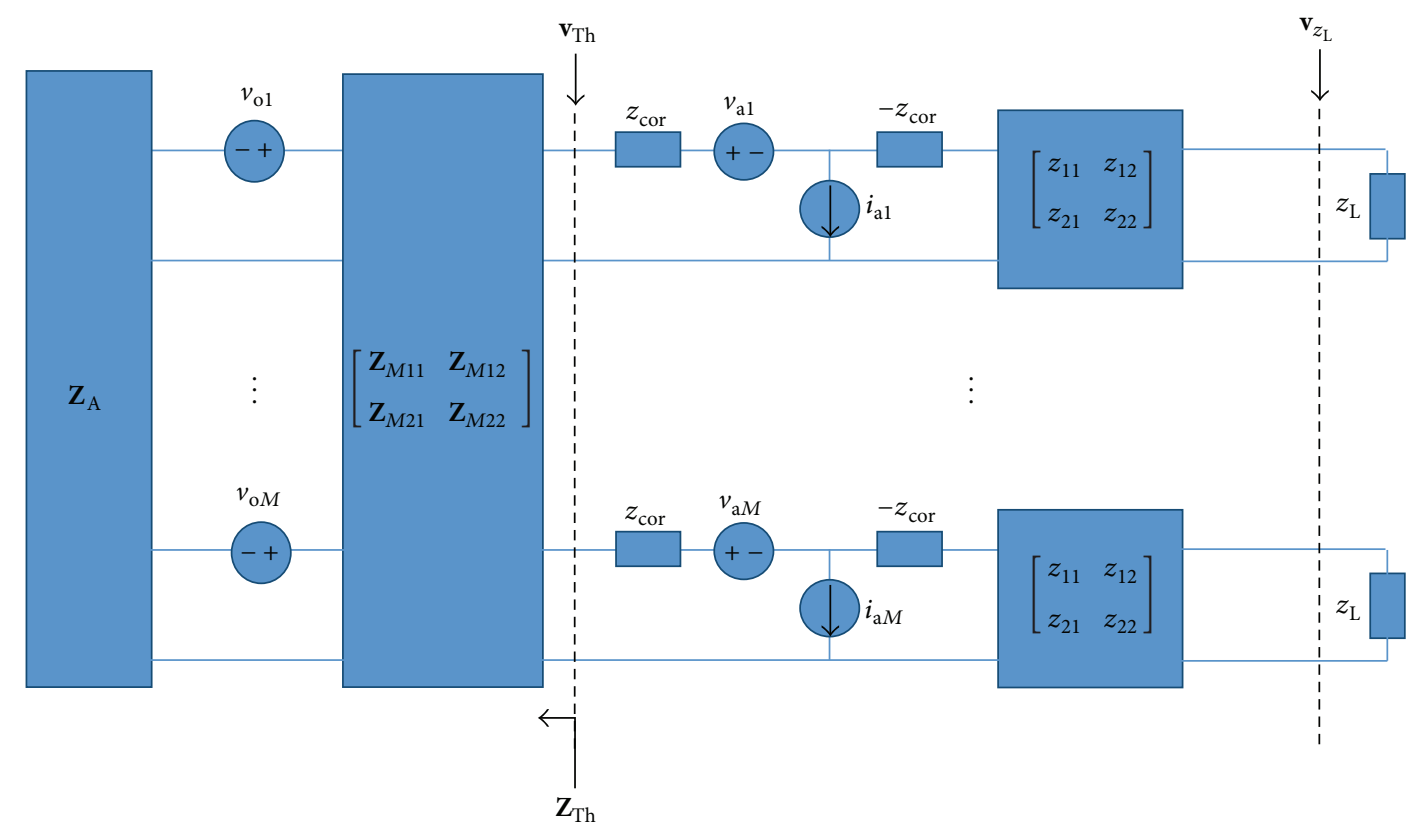

FIGURE 1: Receiver circuit model. According to Rothe-Dahlke noise model, any two-port circuit with internal noise sources can be represented by a $2 \times 2$ equivalent network impedance matrix, noise voltage, and current sources $v_{\mathrm{a} m}, i_{\mathrm{a} m}$, and correlation impedance $z_{\mathrm{cor}}$. This figure is derived from Figure 2 of the paper in [14].

correlation impedance [22] shown in Figure 1, and G is a transformation matrix which is diagonal since the receiver channels downstream the matching network are assumed to be independent. As $\mathbf{G}$ is diagonal, it is also invertible. Since $\mathbf{G}$ and $\mathbf{R}_{\mathrm{A}}$ are both invertible matrices and given that invertible transformations do not influence the capacity of a communications link [1, pp. 183-241], it is convenient to normalize $\mathbf{v}_{z_{\mathrm{L}}}$ in order to obtain a simpler expression as

$$
\mathbf{v}_{\mathrm{L}} \triangleq\left(\frac{\mathbf{R}_{\mathrm{A}}^{1 / 2}}{\sqrt{r_{0}}}\right) \mathbf{G}^{-1} \mathbf{v}_{\mathrm{z}_{\mathrm{L}}}=s \mathbf{h}_{\mathrm{S}}+\mathbf{v}_{\mathrm{N}},
$$

where $\mathbf{v}_{\mathrm{N}}=\mathbf{v}_{\mathrm{EN}}-\left(\mathbf{R}_{\mathrm{A}}^{1 / 2} / \sqrt{r_{0}}\right) \mathbf{v}_{\mathrm{IN}}$ represents the overall system noise and $\mathbf{h}_{\mathrm{S}}, \boldsymbol{s}$ were defined earlier. Note that the normalization in (3) maps the load voltages $\mathbf{v}_{z_{\mathrm{L}}}$ to the opencircuited antenna terminal voltages $\mathbf{v}_{\mathrm{L}}$.

Recall that $\mathbf{v}_{\mathrm{a}}$ and $\mathbf{i}_{\mathrm{a}}$ are mutually independent and that the front-end amplifiers and the downstream receiver components are identical and uncoupled. As such, the intrinsic noise covariance matrix is diagonal; that is, $\mathrm{E}_{\mathbf{v}_{\text {IN }}}\left[\mathbf{v}_{\mathrm{IN}} \mathbf{v}_{\mathrm{IN}}^{H}\right]=$ $\sigma_{\text {IN }}^{2}$ I. Consequently, the overall system noise covariance may be given as

$$
\mathbf{Q}_{\mathrm{N}}=\mathrm{E}_{\mathbf{v}_{\mathrm{N}}}\left[\mathbf{v}_{\mathrm{N}} \mathbf{v}_{\mathrm{N}}^{H}\right]=\mathbf{Q}_{\mathrm{EN}}+\frac{\sigma_{\mathrm{IN}}^{2}}{r_{0}} \mathbf{R}_{\mathrm{A}},
$$

where $\mathbf{Q}_{\mathrm{EN}}=\mathrm{E}_{\mathbf{v}_{\mathrm{EN}}}\left[\mathbf{v}_{\mathrm{EN}} \mathbf{v}_{\mathrm{EN}}^{H}\right]$ is the covariance of the EN noise.

\section{Channel Capacity with Multiport Matching}

A SIMO channel is considered and channel state information (CSI) is assumed to be known at the receiver. That is, the vector of open-circuit instantaneous complex channel gains, $\mathbf{h}_{\mathrm{S}}$, and the covariance of the zero-mean CN noise, $\mathbf{Q}_{\mathrm{N}}$, are known and are constant during a signal snapshot period, which is defined here as the time duration for transmission and reception of one information symbol.

The objective of the receiver processing is that of the estimation of the transmit information symbol $s$, based on combining the channel event observables of $\mathbf{v}_{\mathrm{L}}$, which is referred to as spatial match filtering (SMF) in this paper. Since noise is Gaussian, the optimum SMF processing is a Generalized Matched Filter (GMF). The GMF output is a scalar sufficient statistic given as [23, pp. 524-32]

$$
T=\operatorname{Re}\left(\mathbf{w}^{H} \mathbf{v}_{\mathrm{L}}\right) \text {, }
$$

where $\operatorname{Re}[x]$ is the real part of $x$ and

$$
\mathbf{w}=\frac{\mathbf{Q}_{\mathrm{N}}^{-1} \mathbf{h}_{\mathrm{S}}}{\left\|\mathbf{Q}_{\mathrm{N}}^{-1} \mathbf{h}_{\mathrm{S}}\right\|_{2}}
$$

is the normalized vector of optimum SMF weights. Also, $\|\mathbf{x}\|_{2}$ is the Euclidean norm of $\mathbf{x}$.

Information capacity, or simply capacity, is defined as the supremum of the achievable data rate, at which the transmit symbol, $s$, can be reconstructed at the receiver with arbitrarily low probability of error [1, pp. 183-241]. Let a channel event be defined as the process of transmission and reception of a single information symbol. For a continuous alphabet $\mathrm{CN}$ channel, the capacity in bits-per-transmission (bpt) is given by Shannon's relation as

$$
C=\log _{2}(1+\eta)
$$


where $\eta$ is SNR. Since the SMF processing of (5) preserves mutual information, it achieves the capacity of the SIMO channel. The SMF output SNR may be given as

$$
\eta=\frac{P_{\mathrm{S}}}{P_{\mathrm{N}}}=\frac{\varepsilon \mathbf{w}^{H} \mathbf{h}_{\mathrm{S}} \mathbf{h}_{\mathrm{S}}^{H} \mathbf{w}}{\mathbf{w}^{H} \mathbf{Q}_{\mathrm{N}} \mathbf{w}},
$$

where $P_{\mathrm{S}}=\left|\mathrm{E}_{\mathbf{v}_{\mathrm{N}}}[T \mid s]\right|^{2}=\mathcal{E} \mathbf{w}^{H} \mathbf{h}_{\mathrm{S}} \mathbf{h}_{\mathrm{S}}^{H} \mathbf{w}$ and $P_{\mathrm{N}}=\mathrm{E}_{\mathbf{v}_{\mathrm{N}}}\left[T T^{*} \mid\right.$ $s]-\left|E_{\mathbf{v}_{\mathrm{N}}}\left[\begin{array}{ll}T & \mid\end{array}\right]\right|^{2}=\mathbf{w}^{H} \mathbf{Q}_{\mathrm{N}} \mathbf{w}$ represent signal and noise energies, respectively. The operation $\mathrm{E}_{\mathrm{x}}[a \mid b]$ denotes the expectation of " $a$ " conditioned on " $b$ " with respect to the random properties of " $\mathbf{x}$." $\mathcal{E}$ is the transmit symbol energy defined earlier. The capacity relation of (7) is known as the instantaneous capacity or the capacity of the time-invariant channel. In scattering environments, where $\mathbf{h}_{\mathrm{S}}$ is a random fading process, a long term rate of reliable communications in bits-per-transmission (bits-per-transmission is equivalent to $\mathrm{bits} / \mathrm{sec} / \mathrm{Hz}$ when intersymbol interference is negligible) may be obtained by averaging over many realizations of the fading channel as

$$
\bar{C}=\mathrm{E}_{\mathbf{h}_{\mathrm{S}}}\left[\log _{2}(1+\eta)\right] .
$$

Note that capacity relations (7) and (9) are only valid for the assumptions given, namely, that the noise sources are $\mathrm{CN}$ and independent of the transmitter symbol amplitude modulation which is also CN. Clearly, practical source symbol modulations are only approximately $\mathrm{CN}$ which implies a decrease in the source entropy and hence the attained capacity. The justification for the approximations necessary to use Shannon's capacity relation is the profound simplicity of the relation and the fact that it varies monotonically with SNR. Had we considered defining the channel based on a binary transmitter entropy source, then Shannon's capacity relation would no longer be valid and only numerical capacity computations would have been possible. Further considerations of the potential capacity loss due to suboptimal coding schemes and intersymbol interference may be found in [24, pp. 597-688] and are beyond the scope of this paper.

3.1. Dominant Intrinsic Noise. When noise in the system is predominantly from the noisy receiver electronics, for example, from receiver front-end LNAs, the noise covariance of (4) may be approximated as $\mathbf{Q}_{\mathrm{N}} \approx\left(\sigma_{\mathrm{IN}}^{2} / r_{0}\right) \mathbf{R}_{\mathrm{A}}$. Subsequently, the instantaneous capacity of the SIMO system with dominant IN can be found by replacing this approximate noise covariance in (8) and (7) becomes

$$
C_{\mathrm{IN}}=\log _{2}\left(1+\frac{r_{0}}{R_{11}} \frac{\varepsilon}{\sigma_{\mathrm{IN}}^{2}} G_{\mathrm{A}} G_{\mathrm{MC}}\right),
$$

where $R_{11}$ denotes the dipole self-resistance and

$$
G_{\mathrm{A}}=\mathbf{w}^{H} \mathbf{h}_{\mathrm{S}} \mathbf{h}_{\mathrm{S}}^{H} \mathbf{w}
$$

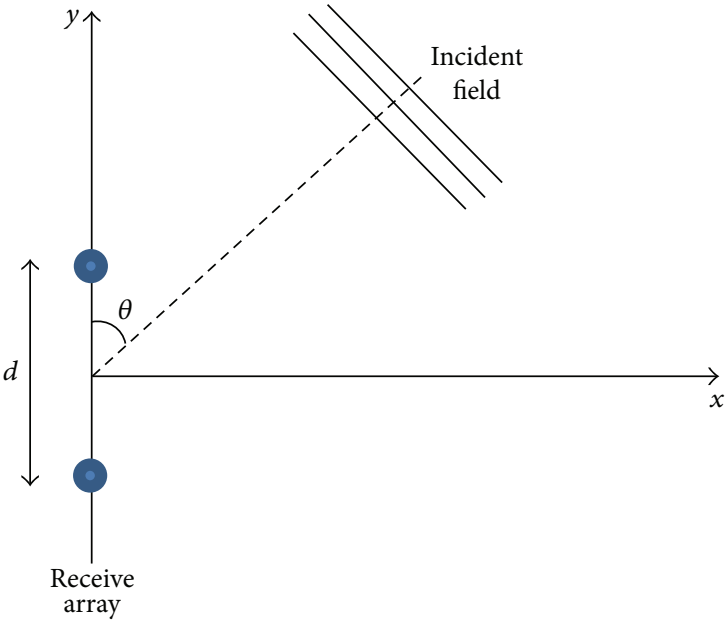

FIgURE 2: SIMO system with two identical center-fed dipoles arranged side by side on the horizontal plane $(x y)$ at the receiver side. The dipoles are aligned in the direction of the $z$-axis and are copolarized with the incident electric field which is also linearly polarized.

represents the aperture gain which is defined here as the gain in the signal power collection capability of the MENIA system relative to the single channel receiver (SISO). Also,

$$
G_{\mathrm{MC}}=\frac{R_{11}}{\mathbf{w}^{H} \mathbf{R}_{\mathrm{A}} \mathbf{w}}
$$

quantifies the SNR gain due to antenna mutual coupling. For the MENIA with $\mathbf{R}_{\mathrm{A}}=R_{11} \mathbf{I}$ the mutual coupling gain is unity; that is, $G_{\mathrm{MC}}=1$.

Next, we will consider a simple SIMO architecture based on a receiver MEA made from a pair of electrically short dipoles in free space to further simplify (10) and derive closed-form expressions that reveal the underlying mechanisms of capacity enhancement based on closely spaced antennas under dominant IN conditions.

3.1.1. The Two-Element MEA in Free Space. Consider a receive MEA consisting of two identical electrically short dipoles in free space arranged side by side on the horizontal plane $(x y$ plane) with a spacing $d$, measured in wavelengths, as shown in Figure 2. The pair of dipoles are assumed to be subjected to a signal plane wave excitation arriving from the direction identified by angle $\theta$.

First, consider that the incident signal is arriving from a direction normal to the array, that is, the broadside case (BS) with $\theta=90^{\circ}$ in Figure 2. Due to the symmetry of this configuration the induced antenna currents are in-phase and equal in magnitude; hence the optimum receiver processing is to trivially add the receiver output observables. That is, $\mathbf{h}_{\mathrm{S}}=$ $[1,1]^{\dagger}$, and from $(6) \mathbf{w}=(1 / \sqrt{2})[1,1]^{\dagger}$, where " $\dagger$ " denotes the matrix transpose. The aperture gain may then be given from (11) as $G_{\mathrm{A}}=2$, which is independent of antenna spacing, $d$. Also, since $\mathbf{w}$ is an eigenvector of $\mathbf{Q}_{\mathrm{N}} \approx\left(\sigma_{\mathrm{IN}}^{2} / r_{0}\right) \mathbf{R}_{\mathrm{A}} \in \mathbb{R}^{2 \times 2}$, the mutual coupling gain may be found from (12) as $G_{\mathrm{MC}}=$ $R_{11} / \lambda_{\mathbf{w}}\left(\mathbf{R}_{\mathrm{A}}\right)$, where $\lambda_{\mathbf{w}}\left(\mathbf{R}_{\mathrm{A}}\right)=R_{11}+R_{12}$ is the eigenvalue 


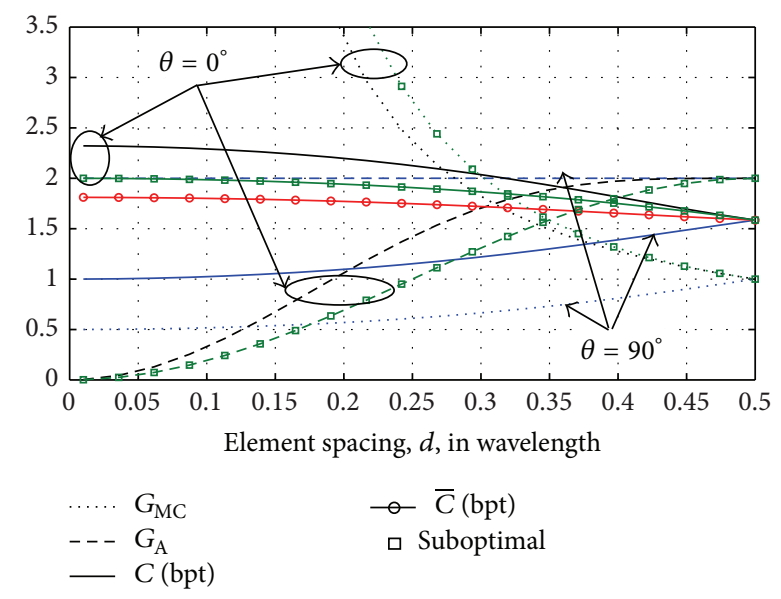

FIGURE 3: Aperture gain $\left(G_{\mathrm{A}}\right)$, mutual coupling gain $\left(G_{\mathrm{MC}}\right)$, instantaneous capacity $(C)$, and mean capacity $(\bar{C})$ versus antenna spacing $d$, for a SIMO system comprising two electrically short receive dipoles. All curves are based on a sample SISO SNR of zero dB. The graphs marked by “ $\square$ " are produced based on the suboptimal SMF processing with $\mathbf{w}=(1 / \sqrt{2})[-1,1]^{\dagger}$.

corresponding to $\mathbf{w}$ and $R_{12}$ denotes the mutual resistance between the two receive antennas. Consequently, the capacity of the broadside MEA $\left(\theta=90^{\circ}\right.$ in Figure 2$)$ under dominant IN conditions may be derived from (10) as

$$
C_{\mathrm{IN}}^{\mathrm{BS}}=\log _{2}\left(1+\frac{r_{0}}{R_{11}} \frac{\varepsilon}{\sigma_{\mathrm{IN}}^{2}} \frac{2}{\left(1+R_{12} / R_{11}\right)}\right) .
$$

From (13), as $d$ approaches zero, $R_{12} \rightarrow R_{11}$ and $G_{\mathrm{MC}}$ approaches 0.5 , which implies an equivalent loss of SNR by a factor of two relative to the MENIA system where $R_{12}=0$. This observation is consistent with the generally accepted notion that larger values of mutual coupling result in reduced channel capacity. Intuitively, under broadside signal and dominant IN conditions the SIMO system reduces to SISO as antenna spacing approaches zero. This concept is illustrated by the graphs corresponding to $\theta=90^{\circ}$ in Figure 3 , which are calculated from (13) for a sample SISO SNR of $\varepsilon / \sigma_{\text {IN }}^{2}=0 \mathrm{~dB}$.

Next consider that the signal plane wave is arriving from the direction of the array, that is, the endfire case (EF) with $\theta=0^{\circ}$ in Figure 2. The normalized vector of channel gains for the endfire MEA may be expressed as $\mathbf{h}_{\mathrm{S}}=\left[e^{j \pi d}, e^{-j \pi d}\right]^{\dagger}$. Unfortunately for the endfire case the capacity relation based on the optimal SMF processing cannot be expressed in closed form. Here we consider a suboptimal SMF processing based on $\mathbf{w}=(1 / \sqrt{2})[-1,1]^{\dagger}$ to simplify the algebraic analysis. As will be shown, the results based on this suboptimal processing closely agree with those computed numerically based on the optimum weights vector of (6).

For $\mathbf{w}=(1 / \sqrt{2})[-1,1]^{\dagger}$ the aperture gain may be derived from (11) as $G_{\mathrm{A}}=2 \sin ^{2}(\pi d)$, and the mutual coupling gain from (12) is $G_{\mathrm{MC}}=1 /\left(1-R_{12} / R_{11}\right)$. Consequently, the SIMO

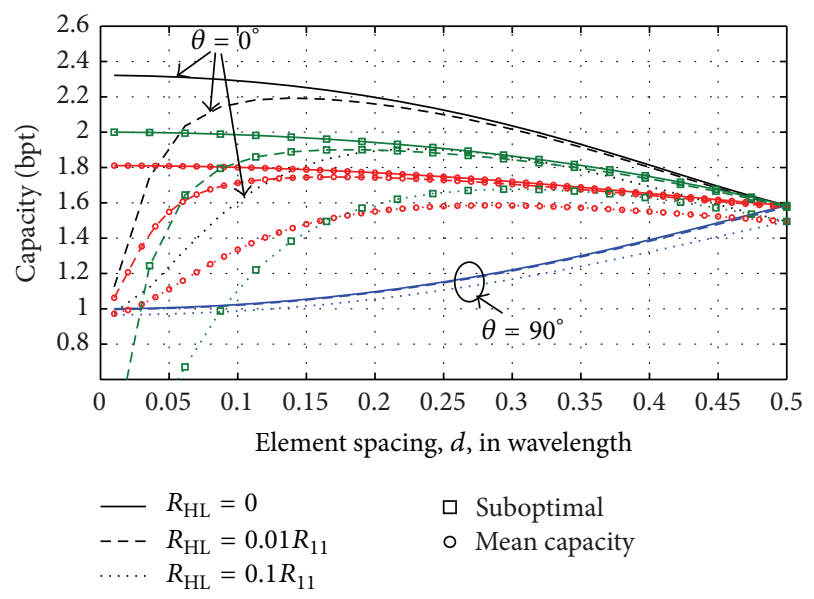

FIGURE 4: Instantaneous and mean capacity versus antenna spacing for a two-element receive MEA with heat losses for an equivalent SISO SNR of zero dB.

capacity of the endfire MEA $\left(\theta=0^{\circ}\right.$ in Figure 2) under dominant IN may be given from (10) as

$$
C_{\mathrm{IN}}^{\mathrm{EF}}=\log _{2}\left(1+\frac{r_{0}}{R_{11}} \frac{\varepsilon}{\sigma_{\mathrm{IN}}^{2}} \frac{2 \sin ^{2}(\pi d)}{\left(1-R_{12} / R_{11}\right)}\right) .
$$

Graphs of $G_{\mathrm{A}}, G_{\mathrm{MC}}$, and $C$ are shown in Figure 3 versus spacing for the endfire case $\left(\theta=0^{\circ}\right)$. These graphs are calculated from (14) for the suboptimal processing, described above, as well as from (10) to (12), based on the optimum weights vector of (6). As indicated by the $G_{\mathrm{A}}$ graphs of Figure 3, the aperture gain decreases to small values as $d$ approaches zero, which implies that the ability of the endfire MEA to capture signal energy degrades when the antenna spacing $d$ is decreased. This decrease in the aperture gain $G_{\mathrm{A}}$ may be attributed to the decreased spatial correlation between the receiver eigenchannel $\mathbf{w}=(1 / \sqrt{2})[-1,1]^{\dagger}$ and the vector of channel gains $\mathbf{h}_{\mathrm{S}}$ (the signal subspace) at small spacings. In other words, as the spacing is decreased to small values, the vector of channel gains and the receiver eigenchannel become perpendicular; that is, $\mathbf{h}_{\mathrm{S}}=\lim _{d \rightarrow 0}\left[e^{j \pi d}, e^{-j \pi d}\right]^{\dagger}=$ $[1,1]^{\dagger}$; hence $\lim _{d \rightarrow 0}\left|\mathbf{w}^{H} \mathbf{h}_{\mathrm{S}}\right|=0$ for $\mathbf{w}=(1 / \sqrt{2})[-1,1]^{\dagger}$. As indicated by the $G_{\mathrm{MC}}$ graphs of Figure 4 , the mutual coupling gain becomes very large when the spacing is decreased to small values. Basically, a smaller spacing results in increased mutual coupling which subsequently further correlates the channel noise at the open-circuited antenna terminals (recall that the normalization that resulted in (3) maps all voltages to the open-circuited antenna terminals, $\mathbf{v}_{\mathrm{L}}$ ). This increased noise correlation, which may be quantified by the ratio $R_{12} / R_{11}$, is then exploited by the SMF processing to attenuate the noise power in the receiver eigenchannel. That is, since $\mathbf{w}=(1 / \sqrt{2})[-1,1]^{\dagger}$ is the eigenvector that corresponds to the smallest eigenvalue of the noise covariance matrix, $\mathbf{Q}_{\mathrm{N}}=$ $\left(\sigma_{\mathrm{IN}}^{2} / r_{0}\right) \mathbf{R}_{\mathrm{A}}$, the noise power of the receiver eigenchannel from (8) is $P_{\mathrm{N}}=\mathbf{w}^{H} \mathbf{Q}_{\mathrm{N}} \mathbf{w}=\lambda_{\mathbf{w}}\left(\mathbf{Q}_{\mathrm{N}}\right)=R_{11}-R_{12}$, which approaches zero as $\mathbf{Q}_{\mathrm{N}}$ becomes singular and $R_{12} \rightarrow$ $R_{11}$. Consequently, $G_{\mathrm{MC}}$ which is inversely proportional to 
$P_{\mathrm{N}}$ becomes infinite as the spacing $d$ approaches zero. The SMF output SNR $\eta$, however, remains finite as the product $G_{\mathrm{A}} G_{\mathrm{MC}}$ levels off at a value that for $d \approx 0$ is about $3 \mathrm{~dB}$ larger than the SMF output SNR at $d=0.5$. The interesting result that $G_{\mathrm{A}} G_{\mathrm{MC}}$ remains finite even with $d$ approaching zero is tantamount to the fact that "theoretically there is no limit to the directivity of a linear array" [25, pp. 306-7]. As indicated by the $C$ graphs corresponding to $\theta=0^{\circ}$ in Figure 3, a net capacity gain of up to about $1 \mathrm{bpt}$ may be realized at very small spacings relative to $d=0.5$. Note that the formula $R_{12}=R_{11} \operatorname{sinc}(2 \pi d)$ is used to calculate the value of mutual resistance between the two electrically short dipoles. Therefore, $d=0.5$ corresponds to the MENIA system with zero mutual coupling.

The fact that an increased channel capacity can result from increasing mutual coupling between the MEA elements is an important observation which contradicts the generally accepted notion that mutual coupling invariably negatively impacts channel capacity. Next, we will extend our analysis to arbitrary MEAs made from $M$ electrically short antennas.

3.1.2. The Arbitrary MEA. When antenna spacing is decreased to small values, mutual coupling between MEA elements invariably increases, which subsequently further correlates the intrinsic channel noise. As will be shown in Section 3.2.2, when channel noise is highly correlated the optimum SMF processing is to steer the receiver eigenchannel $\mathbf{w}$ in a spatial direction which is orthogonal to the eigenvector corresponding to the largest eigenvalue of the noise covariance matrix $\mathbf{Q}_{\mathrm{N}}$, provided that such perpendicular mapping is not perpendicular also to the signal subspace. As antenna spacings approach zero, $\mathbf{Q}_{\mathrm{N}}$ becomes singular; hence, the noise power in the receiver eigenchannel approaches zero; that is, $P_{\mathbf{N}}=\mathbf{w}^{H} \mathbf{Q}_{\mathrm{N}} \mathbf{w} \rightarrow 0$. Significant SNR gain may be achieved if the signal subspace remains at least partially spatially correlated to the receiver eigenchannel, that is, the dot-product $\left|\mathbf{w}^{H} \mathbf{h}_{\mathrm{S}}\right|>0$ such that $P_{\mathrm{S}}=\varepsilon \mathbf{w}^{H} \mathbf{h}_{\mathrm{S}} \mathbf{h}_{\mathrm{S}}^{H} \mathbf{w}>0$. This condition is equivalent to realizing superdirectivity for the closely spaced antenna array. In this context superdirectivity may be interpreted as the improvement in the ability of the antenna array to attenuate noise while maintaining a relatively large gain in the direction of the signal subspace.

Our understanding of the fundamental mechanisms of capacity enhancement with closely spaced antennas in free space may be extended to include scattering scenarios as follows. Note that full CSI implies that the dyadic product $\mathbf{h}_{\mathrm{S}} \mathbf{h}_{\mathrm{S}}^{H}$ always results in a rank-one dyad, which applies to arbitrary signal channel gains due to free space as well as those due to scattering. Therefore, the optimum SMF processing formulas (5), (6), and (8) and the instantaneous capacity relation (7) apply to free space as well as scattering cases.

The effect of antenna spacing on the capacity of the twoelement MEA depicted in Figure 2 under Rayleigh fading conditions is illustrated by the $\bar{C}$ curve in Figure 3. This graph is computed from (9) based on averaging over 10,000 independent realizations of the fading channel for each antenna spacing. As indicated by $\bar{C}$ graph of Figure 3 , the long term rate of reliable communications improves when element spacing decreases. That is, the mean capacity of the MECSA system with mutual coupling is larger than the mean capacity of the MENIA system for $d=0.5$. Note that since superdirectivity is only realizable for those realizations of $\mathbf{h}_{\mathrm{S}}$ that are highly correlated with the spatial direction of array's endfire, the mean capacity is smaller than the instantaneous capacity of the endfire case as indicated by their corresponding graphs in Figure 3.

3.2. Extrinsic Noise. The capacity of MECSA system under dominant EN conditions will be treated in this section. Two scenarios involving dominant spatially white interference (SWI) and dominant HDI will be considered.

3.2.1. Spatially White Interference. In rich scattering environments, such as inside buildings, EN is commonly modeled in three dimensions as spatially white plane wave processes generated by isotropic sources that are located on the surface of a sphere whose radius is very large comparing to the physical dimensions of the MEA and the radiated signal wavelength (the isotropic sphere model) [17, pp. 352-64]. As shown in [26, pp. 593-4], the normalized matrix of antennas mutual resistances and the normalized covariance of the EN due to SWI are identical (i.e., $\mathbf{R}_{\mathrm{A}} \propto \mathbf{Q}_{\mathrm{EN}}$ ) for minimum scattering antennas, which includes the electrically short dipole considered in this paper as a special case. Thus the results of Section 3.1 are readily applicable to equivalent MEA systems that are under dominant SWI. However, the capacity enhancement under SWI conditions is due to the spatially correlated EN at open-circuit antenna terminals, not to mutual coupling as evident from (3). Intuitively, both signal and EN are subject to the same antenna coupling; hence, mutual coupling does not impact channel capacity under dominant EN conditions.

3.2.2. Highly Directional Interference. In this section, the example of a single interferer will be considered to illustrate how antenna spacing influences channel capacity under HDI conditions. The results of this section can be readily extended to multiple interferers by noting that the optimum SMF weights vector for the multiple interferer case is the weighted sum of the SMF weights vectors corresponding to the individual interfering signals [17, pp. 452-72].

Consider the $M$-element linear MEA of Figure 1. Let the total channel noise involve two independent noise processes: (1) a strong HDI component represented by the normalized spatial basis vector $\mathbf{h}_{\mathrm{HDI}} \in \mathbb{C}^{M}$, where $\mathbf{h}_{\mathrm{HDI}}^{H} \mathbf{h}_{\mathrm{HDI}}=M$; let $\varepsilon_{\mathrm{HDI}}$ denote the interference power delivered to the load $z_{\mathrm{L}}$ of a signal antenna receiver; (2) a relatively weak White Gaussian Noise (WGN) process with the density $\mathrm{CN}\left(0, \sigma_{0}^{2} \mathbf{I}\right)$. This WGN process is primarily considered to facilitate the matrix inversion associated with the derivation of the optimum SMF weights vector $\mathbf{w}$ from (6). Dominant HDI implies that $\varepsilon_{\text {HDI }} \gg \sigma_{0}^{2}$.

The total channel noise covariance may be expressed as

$$
\mathbf{Q}_{\mathrm{N}}=\sigma_{0}^{2} \mathbf{I}+\varepsilon_{\mathrm{HDI}} \mathbf{h}_{\mathrm{HDI}} \mathbf{h}_{\mathrm{HDI}}^{H} .
$$


As before, the optimum SMF weights vector may be found by replacing (15) in (6) as

$$
\begin{aligned}
\mathbf{w} & =\frac{1}{\left\|\mathbf{Q}_{\mathrm{N}}^{-1} \mathbf{h}_{\mathrm{S}}\right\|_{2}} \mathbf{Q}_{\mathrm{N}}^{-1} \mathbf{h}_{\mathrm{S}} \\
& =\frac{1}{\left\|\mathbf{Q}_{\mathrm{N}}^{-1} \mathbf{h}_{\mathrm{s}}\right\|_{2}}\left(\sigma_{0}^{2} \mathbf{I}+\varepsilon_{\mathrm{HDI}} \mathbf{h}_{\mathrm{HDI}} \mathbf{h}_{\mathrm{HDI}}^{H}\right)^{-1} \mathbf{h}_{\mathrm{S}} \\
& =c\left(\mathbf{I}-\frac{\varepsilon_{\mathrm{HDI}}}{\sigma_{0}^{2}+M \varepsilon_{\mathrm{HDI}}} \mathbf{h}_{\mathrm{HDI}} \mathbf{h}_{\mathrm{HDI}}^{H}\right) \mathbf{h}_{\mathrm{S}},
\end{aligned}
$$

where Woodbury's identity for matrix inversion is used, $\mathbf{h}_{\mathrm{S}} \epsilon$ $\mathbb{C}^{M}$ is the vector of channel gains, and $c$ is a normalizing scalar such that $\mathbf{w}^{H} \mathbf{w}=1$. When HDI is dominant, $\boldsymbol{E}_{\mathrm{HDI}} \gg \sigma_{0}^{2}$ such that (16) can be approximated as

$$
\mathbf{w} \approx c\left(\mathbf{I}-\frac{\mathbf{h}_{\mathrm{HDI}} \mathbf{h}_{\mathrm{HDI}}^{H}}{M}\right) \mathbf{h}_{\mathrm{S}} .
$$

By taking a closer look at (17), one realizes that the term within the brackets is the projection matrix onto the subspace orthogonal to $\mathbf{h}_{\mathrm{HDI}}$; that is, $\mathbf{w}^{H} \mathbf{h}_{\mathrm{HDI}}=0$. That is, the optimum SMF processing is to steer the one-dimensional receiver eigenchannel in the direction that is orthogonal to the interference subspace. Under LOS conditions, this processing is tantamount to forming a deep null in the direction of the incoming interference. For the scattering case, this is equivalent to putting the interference signal in a deep fade.

The instantaneous SIMO capacity may be found by replacing $\mathbf{w}$, from (17), in (8) and (7) becomes

$$
\begin{aligned}
& C_{\mathrm{HDI}} \\
& \quad=\log _{2}\left[1+\mathcal{E} M\left(1+\frac{\varepsilon_{\mathrm{HDI}}}{\sigma_{0}^{2}}\right)\left(1-\rho^{2}\left(\mathbf{h}_{\mathrm{S}}, \mathbf{h}_{\mathrm{HDI}}\right)\right)\right],
\end{aligned}
$$

where $\rho\left(\mathbf{h}_{\mathrm{S}}, \mathbf{h}_{\mathrm{HDI}}\right) \triangleq\left|\mathbf{h}_{\mathrm{HDI}}^{H} \mathbf{h}_{\mathrm{S}}\right| / M$ represents the normalized spatial correlation coefficient between the signal and HDI subspaces. As the antenna spacings approach zero, signal and HDI subspaces blend together such that $\rho\left(\mathbf{h}_{\mathrm{S}}, \mathbf{h}_{\mathrm{HDI}}\right) \rightarrow 1$, which subsequently results in a significant capacity loss as can be seen from (18). Intuitively, as the spatial resolution of the antenna system degrades with smaller antenna spacings, the ability of the MEA system to adequately separate signal from interference decreases, hence the observed capacity loss.

3.3. Heat Losses in the Antenna System. Thus far, the array elements were assumed to be made from perfectly conducting wires. Practical antennas, however, are lossy; for example, they are made from materials with finite conductivity, such that some of the absorbed power appears in the form of heat in the antenna conductor, insulator, and so forth. The question of heat losses (HL) and their effect on the capacity of the densely packed array will be treated in this section.

As the electrically short antenna is assumed with fixed current distribution that only scales in amplitude, heat losses can be modeled by a simple series loss resistor $R_{\mathrm{HL}}$ at the antenna terminal [27, pp. 548-52]. That is, the total terminal resistance of an isolated antenna may be given as the sum of self-resistance $R_{11}$ and the equivalent loss resistance $R_{\mathrm{HL}}$. The heat losses in the MEA, comprising identical electrically short lossy antennas, can be modeled by replacing the mutual resistance matrix $\mathbf{R}_{\mathrm{A}}$ with $\mathbf{R}_{\mathrm{A}}+R_{\mathrm{HL}} \mathbf{I}$.

Consider the simple example of the two-element receive MEA introduced earlier in Figure 2. Under dominant IN conditions approximate closed-form expressions for the channel capacity can be obtained by replacing $R_{11}$ with $R_{11}+R_{\mathrm{HL}}$ in (13) as

$$
\begin{aligned}
& C_{\mathrm{IN}}^{\mathrm{BS}, \mathrm{HL}} \\
& \quad=\log _{2}\left[1+\frac{r_{0}}{R_{11}+R_{\mathrm{HL}}} \frac{\varepsilon}{\sigma_{\mathrm{IN}}^{2}} \frac{2}{\left(1+R_{12} /\left(R_{11}+R_{\mathrm{HL}}\right)\right)}\right]
\end{aligned}
$$

for the broadside case and replacing in (14) gives the capacity of the endfire case as

$$
\begin{aligned}
& C_{\mathrm{IN}}^{\mathrm{EF}, \mathrm{HL}} \\
& \quad=\log _{2}\left[1+\frac{r_{0}}{R_{11}+R_{\mathrm{HL}}} \frac{\varepsilon}{\sigma_{\mathrm{IN}}^{2}} \frac{2 \sin ^{2}(\pi d)}{\left(1-R_{12} /\left(R_{11}+R_{\mathrm{HL}}\right)\right)}\right] .
\end{aligned}
$$

The effect of antenna heat losses on the channel capacity of the two-element MEA is illustrated by the capacity-versusspacing curves in Figure 4 for three cases of $R_{\mathrm{HL}} / R_{11}=$ $0,0.01$, and 0.1 . The instantaneous capacity graphs for $\theta=90^{\circ}$ and $\theta=0^{\circ}$ are calculated from (19) and (20), respectively. As indicated by the $\theta=90^{\circ}$ graphs, capacity of the broadside MEA is only marginally influenced by the heat losses in the antenna system. Comparing (19) and (13) shows that when $R_{\mathrm{HL}} / R_{11} \ll 1$, then $C_{\mathrm{IN}}^{\mathrm{BS}, \mathrm{HL}} \approx C_{\mathrm{IN}}^{\mathrm{BS}}$ which is true for the typical high-frequency antennas with large radiation resistance to loss resistance ratios.

The capacity of the endfire MEA is more dramatically impacted by heat losses in the antenna system, as is evident from $\theta=0^{\circ}$ graphs in Figure 4. Recall that the aperture gain of the endfire MEA approaches zero as $d$ approaches zero. However, unlike the $R_{\mathrm{HL}}=0$ case where the mutual coupling gain $G_{\mathrm{MC}}$ becomes infinitely large when the spacing is decreased to small values, it can be shown that for $R_{\mathrm{HL}} \neq$ 0 the mutual coupling gain $G_{\mathrm{MC}}$ is upper bounded by $1+$ $R_{11} / R_{\mathrm{HL}}$. Therefore, product $G_{\mathrm{A}} G_{\mathrm{MC}}$ approaches zero as $G_{\mathrm{A}}=$ $2 \sin ^{2}(\pi d)$ approaches zero when spacing $d$ is decreased, which subsequently significantly attenuates the achievable channel capacity at very small spacings.

Graphs representing mean capacity under Rayleigh fading conditions are also shown in Figure 4 for the two-element MEA. It is apparent from these curves that even a relatively small loss resistance seriously limits the achievable channel capacity at very small spacings, while larger loss resistances cause further capacity reduction at relatively greater spacings. As indicated by the graph $\bar{C}$ for $R_{\mathrm{HL}} / R_{11}=0.01$, which is a likely value for the typical high-frequency antenna, capacity peaks at about $d=0.15$ wavelengths and there is a sharp decrease at spacings smaller than 0.1 wavelengths. In addition, we note that capacity is almost constant for spacings 
between 0.15 and 0.35 wavelengths. Although a spacing of $d=0.15$ results in a smaller MEA size, which is favorable for mobile handheld applications that are subject to physical size constraints, nevertheless, the usable channel bandwidth is less at smaller spacings [12]. Therefore, a spacing of 0.35 may be chosen over 0.15 if a wider bandwidth is desired.

Extension to the analysis of arbitrary MECSA consisting of $M$ lossy antennas can be made by noticing that for nonzero $R_{\mathrm{HL}}$ the matrix $\mathbf{R}_{\mathrm{A}}+R_{\mathrm{HL}} \mathbf{I}$ is positive definite. As such, $\mathbf{w}^{H}\left(\mathbf{R}_{\mathrm{A}}+R_{\mathrm{HL}} \mathbf{I}\right) \mathbf{w}>0$ and $G_{\mathrm{MC}}=R_{11} / \mathbf{w}^{H}\left(\mathbf{R}_{\mathrm{A}}+R_{\mathrm{HL}} \mathbf{I}\right) \mathbf{w}$ is upper bounded. Consequently, it can be shown that the product $G_{\mathrm{MC}} G_{\mathrm{A}}$ decreases when spacing is decreased to less than a threshold value (e.g., $d<0.15$ wavelengths for the twoelement MEA) that is larger for bigger $R_{\mathrm{HL}} / R_{11}$.

As a final remark we note that the capacity of the MEA system with dominant EN is not influenced by heat losses in the antenna system since both signal and EN are subject to the same loss resistance at the antenna terminals. This result, however, may not be extended to practical antennas that are spaced very closely, for example, $d<0.1$ wavelengths, since the assumptions of minimum scattering and electrically short are not valid at these spacings.

3.4. Bandwidth of Operation. One of the practical problems associated with superdirective MECSAs is their narrow bandwidth of operation. The bandwidth of operation is defined here as the range of frequencies within which the pattern characteristics of the array (e.g., gain, beam direction, and beamwidth) remain within an acceptable value of those at the center frequency [25, pp. 63-4]. Note that a narrow bandwidth of operation is also tantamount to high sensitivity to fluctuations in the SMF weights vector. The bandwidth of operation is approximately equal to the inverse of array geometrical quality factor $[12,28]$, which for a linear array comprising identical minimum scattering elements can be shown to be identical to the expression for $G_{\mathrm{MC}}$, given in (12). That is, as the achievable SNR increases according to product $G_{\mathrm{A}} G_{\mathrm{MC}}$, the usable bandwidth decreases according to $\left(1 / G_{\mathrm{MC}}\right)$. As shown in Section 3, the mean capacity of the example SIMO system is relatively unchanged when the element spacing is varied in the range of $0.1<d<0.3$ wavelengths. Therefore, when a larger channel bandwidth is desired a spacing of about $d=0.3$ is favorable. From Figure 3, at $d=0.3$, product $G_{\mathrm{A}} G_{\mathrm{MC}}=2.7$, which is about $1.3 \mathrm{~dB}$ larger than the value at $d=0.5$ (MENIA), indicating a net SNR gain. This SNR gain corresponds to a capacity increase of about $0.3 \mathrm{bpt}$ and a reduction in the bandwidth of operation to about $67 \%$ relative to the equivalent MENIA system. The impact of element spacing on the usable bandwidth of MEAs with a large number of elements, $M \gg 2$, will be more pronounced since $G_{\mathrm{MC}}$ will grow faster as the elements are brought closer together.

\section{General Antenna with Load Mismatch}

The previous derivations of SIMO system capacity were simplified based on the assumption that the receive dipoles were made from very thin wires and were electrically short such that the current distribution on the antenna wires was constrained to be a single current basis function. Practical antennas, however, are not electrically short and the current distribution on antenna conductor is influenced by loading and the presence of nearby antennas. In addition, ideal multiport matching networks are difficult to implement and practical MEAs are typically partially mismatched. This partial load mismatch results in a reduced signal power at the receiver loads $[11,19,29]$. The question of suboptimal loading and of the effect of changes in antenna current distribution will be treated in this section. The standard MoM processing will be used to evaluate the impact of antenna spacing on channel capacity in practical implementable antenna systems under dominant IN conditions. Similar findings regarding the impact of antenna spacing on the channel capacity under dominant SWI conditions are anticipated as predicted by the theoretical results of Section 3.2.1.

The MoM is a Least Squares (LS) approximation technique for estimating the current distribution on the surface of an electric conductor which is subjected to some source of excitation. Estimation of antenna currents is made based on dividing the antenna conductor into $N$ computational segments, each with a presumed current distribution known as a current basis function. Given the current basis function, the scattered EM fields can be calculated, algebraically. Consequently, boundary conditions may be enforced such that the superposition of the incident and the scattered fields is zero on the conductor surface (point matching).

While the MoM is generally applicable, the example of two identical center-fed standard dipole antennas, made from finite-thickness wires of radius $10^{-4}$ wavelengths, is considered here. Dipole lengths are adjusted to 0.484 wavelengths to obtain a real self-impedance of $Z_{11} \approx R_{11}=72 \Omega$. The receiver antennas are taken to be directly connected to the front-end amplifiers, as shown in Figure 5. The input impedance of each amplifier is assumed to be matched to the dipole self-impedance of $72 \Omega$. This suboptimal matching technique is sometimes referred to as self-matching $[11,14]$. In practice, self-matching may be achieved using several twoport matching networks [14]; nonetheless, such implementation details are beyond the scope of this paper and will not be discussed here. As loading influences the current distribution on antenna conductors, it needs to be accounted for, which is done by placing an equivalent lumped load at the center mode of each dipole antenna in the MoM, [27, pp. 490-9].

We consider the MEA configuration of Figure 2 for our analysis. Each dipole is divided into $N=20$ computational segments. The antennas are excited by both external and internal sources. The external sources are due to the copolarized incident signal plane waves arriving from a horizontal direction. The internal sources of excitation are due to noise in the receiver electronics. Two simplifying assumptions pertinent to many typical RF low-noise amplifiers (LNAs) are that the amplifier gain is large, and that the LNAs are approximately unilateral; that is, $z_{12} \approx 0$ in Figure 5 . With these assumptions, the noise due to the receiver components downstream front-end amplifiers, for example, mixers and filters, is approximately negligible. As such, the front-end noise characteristics may be approximated by the front-end LNAs. The Rothe-Dahlke impedances and noise parameters 


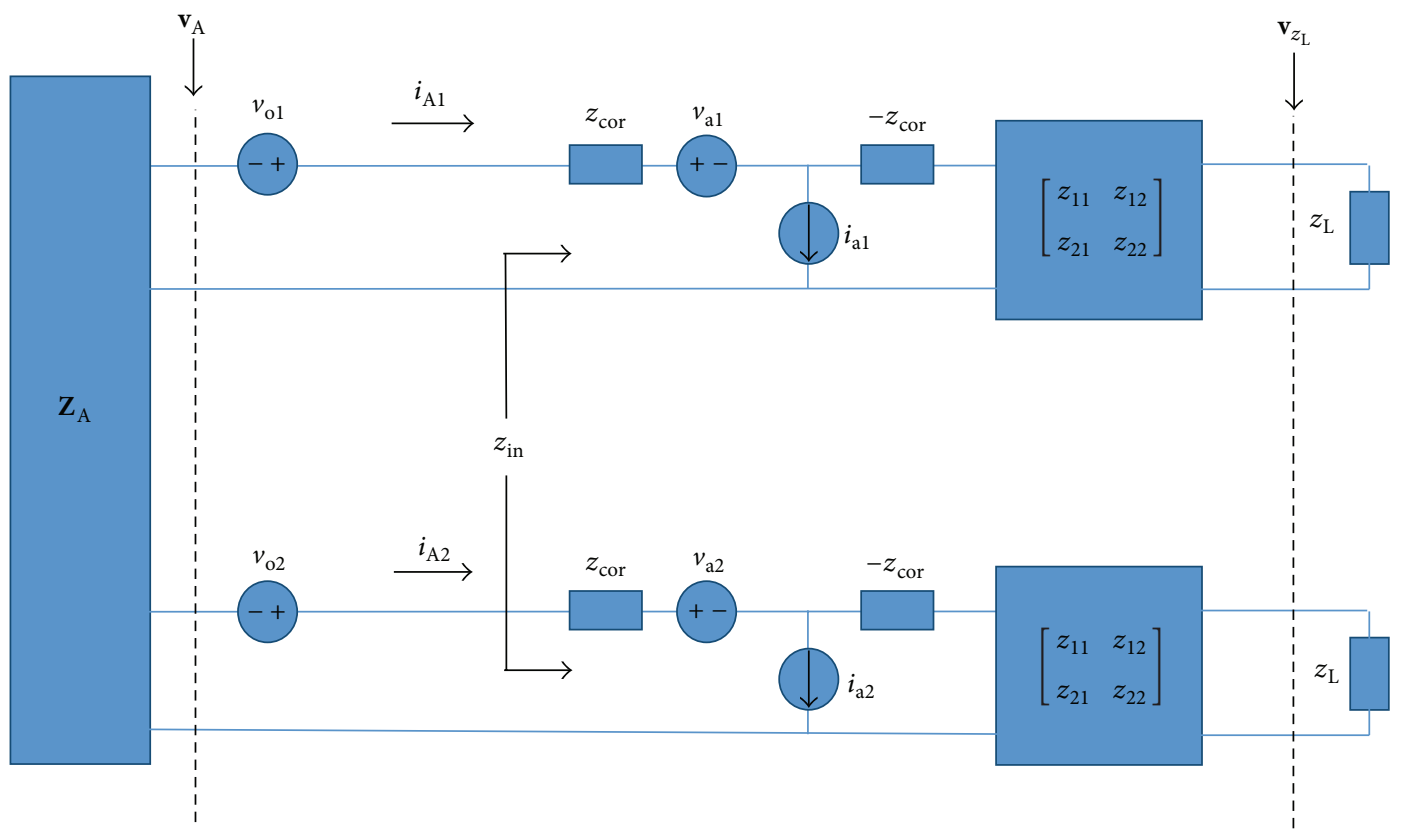

FIGURE 5: Network model for the receive MEA with suboptimal loading.

are taken from the example given in [14], which exemplifies a low-cost LNA used in the $900 \mathrm{MHz}$ frequency range.

A power constraint is applied based on constraining the intensity of the incident signal plane waves to obtain a SISO SNR of $0 \mathrm{~dB}$ at the load, $z_{\mathrm{L}}$. Both the free space and scattering scenarios are considered. For free space, the broadside $\theta=$ $\left.90^{\circ}\right)$ and the endfire $\left(\theta=0^{\circ}\right)$ cases are examined. The scattering scenario is exemplified based on 1000 incident signal plane waves modulated by independent and uniformly distributed phases to typify Rayleigh fading.

The induced antenna terminal currents $\left(i_{\mathrm{A} 1}, i_{\mathrm{A} 2}\right)$ are computed from the MoM and subsequently used to determine signal and noise induced voltages across $z_{\mathrm{L}}$. For each antenna spacing, $d$, the noise covariance, $\mathbf{Q}_{\mathrm{N}}$, is estimated based on averaging over 10,000 independent realizations of the $\mathrm{CN}$ noise samples. Then, the optimum SMF weights are computed from (6) for each realization of the incident signal field. Finally, the SMF output SNR is estimated, from (8), and the instantaneous capacity is calculated from (7).

Representative graphs of channel capacity are given in Figure 6. As can be seen from this figure, the capacity of the broadside MEA decreases monotonically as antenna spacing decreases. This observation is consistent with our theoretical analysis of channel capacity in Section 3.1 and emanates from the fact that superdirectivity is not realizable for broadside arrays. For the endfire case, the capacity peaks at about $d \approx 0.3$ wavelengths. As $d$ decreases, the intrinsic receiver noise becomes correlated due to mutual coupling between the receive antennas, which is subsequently exploited by the SMF processing to produce gain. On the other hand, the antennas become further mismatched to their load impedances, $\mathbf{z}_{\text {in }}$, in Figure 5, as mutual coupling becomes larger. The increasing mismatch losses eventually exceed the realizable gain due to

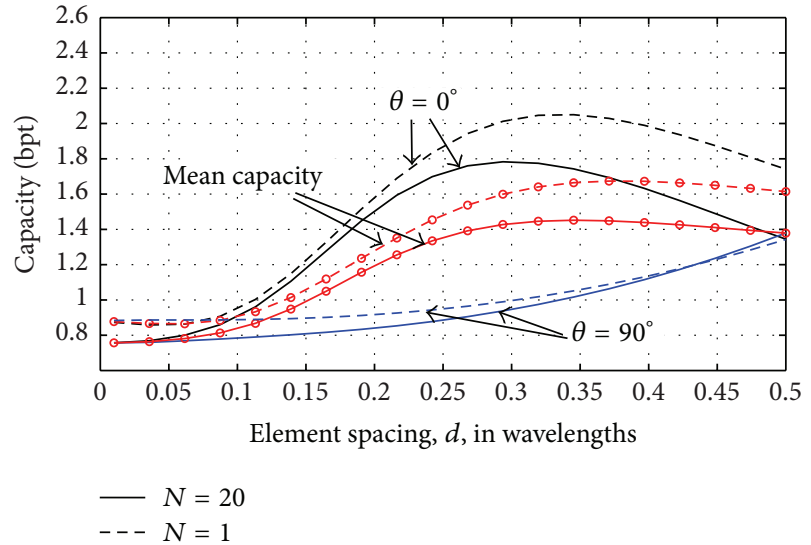

FIGURE 6: Instantaneous and mean capacity versus element spacing for a pair of receive standard dipoles with self-matching. $N$ denotes the number of computational segments on each dipole.

mutual antenna coupling, resulting in the observed capacity loss for $d<0.3$ wavelengths.

The mean capacity graph is also illustrated in Figure 6. As evident from the $\bar{C}$ graph, mean capacity is almost constant (within $0.1 \mathrm{bpt}$ ) for spacings between 0.3 and 0.5 wavelengths. A spacing of 0.3 wavelengths has the advantage of smaller MEA physical size which is a remarkable result as it indicates that a moderate reduction of MEA physical size is possible without having to compromise the achievable channel capacity. We note that smaller MEA sizes may also be realized when a better matching technique, such as the adaptive termination to uncoupled loads [19], is used. 
Next we will constrain the antenna current distributions by setting $N=1$ in the MoM to isolate the effect of changing current distributions from that of load mismatch. By setting $N=1$ we have constrained antenna currents to a single basis function. By comparing the $N=1$ with the $N=20$ graphs of Figure 6, one realizes that the changes in antenna current distribution are not a significant factor in determining the overall capacity of the communications link. Physically, as antenna current distribution further deviates with changes in antenna spacing and loading, its impact on channel capacity is partially neutralized given that both the absorbed signal power and the amount of coupled noise between the MEA elements are affected by such changes.

As a final remark we note that while heat losses in the antenna system are expected to attenuate the achievable capacity, nevertheless, these losses are very small at moderate spacings, for example, at $d=0.3$ for a large class of highfrequency antennas which have radiation resistances that are high relative to a typical loss resistance of about $1 \Omega$. As such, the behavior of moderately compact practical lossy antenna systems may be inferred from the capacity analysis of this section.

An approximation in the analysis is that in the MoM the current is assumed to be constant in azimuth around the wire which is not valid when $d$ is in the order of the wire radius with the exception of $d=0$ for the calculation of the self-impedance. In the presented simulation results the wire radius was $10^{-4}$ wavelengths; therefore, capacity values were not shown for spacings less than 0.01 where the results are less accurate.

\section{Conclusions}

In this paper, we studied the capacity of multiple element closely spaced antenna systems under several scenarios of signal and noise and antenna loading. The analysis shows that channel capacity can, in instances where mutual coupling is considered, be enhanced with optimum setting of antenna spacings. When noise is predominantly intrinsic, mutual coupling between receiver antennas is responsible for capacity enhancement at small element spacings. Under dominant SWI conditions, receiver processing capitalizes on the spatially correlated noise at the open-circuit antenna terminals to improve capacity. For dominant HDI scenarios, the SMF processing is equivalent to steering null(s) in the direction of the interference signal(s). Therefore, unlike the scenarios involving SWI and IN, when the dominating source of interference is highly directional channel capacity cannot be improved by reducing element spacing, since the spatial resolution of the MEA system decreases when antennas are closely spaced. Our analysis shows that heat losses in the antenna system significantly impact capacity at very small spacings, for example, $d<0.2$, while the effect is less pronounced at moderately small spacings, for example, $d>0.35$. Application of the theoretical analysis to the capacity of a SIMO system comprising a pair of standard dipole antennas under suboptimal loading conditions was made based on the standard MoM analysis which shows similar capacity behavior to those predicted by the derived algebraic expressions of channel capacity. It is observed that as current distribution of antenna can change, which was constrained by the electrically short antenna model assumed in our theoretical analysis, the effect of the varying current distribution is not a significant factor in the capacity of practical MEA systems.

\section{Conflict of Interests}

The authors declare that there is no conflict of interests regarding the publication of this paper.

\section{References}

[1] T. M. Cover and J. A. Thomas, Elements of Information Theory, John Wiley \& Sons, 2012.

[2] G. J. Foschini and M. J. Gans, "On limits of wireless communications in a fading environment when using multiple antennas," Wireless Personal Communications, vol. 6, no. 3, pp. 311-335, 1998.

[3] E. A. Jorswieck and H. Boche, "Channel capacity and capacityrange of beamforming in MIMO wireless systems under correlated fading with covariance feedback," IEEE Transactions on Wireless Communications, vol. 3, no. 5, pp. 1543-1553, 2004.

[4] P. N. Fletcher, M. Dean, and A. R. Nix, "Mutual coupling in multi-element array antennas and its influence on MIMO channel capacity," Electronics Letters, vol. 39, no. 4, pp. 342-344, 2003.

[5] S. Lu, H. T. Hui, and M. Bialkowski, "Optimizing MIMO channel capacities under the influence of antenna mutual coupling," IEEE Antennas and Wireless Propagation Letters, vol. 7, pp. 287-290, 2008.

[6] B. N. Getu and R. Janaswamy, "The effect of mutual coupling on the capacity of the MIMO cube," IEEE Antennas and Wireless Propagation Letters, vol. 4, no. 1, pp. 240-244, 2005.

[7] J. X. Yun and R. G. Vaughan, "Multiple element antenna efficiency and its impact on diversity and capacity," IEEE Transactions on Antennas and Propagation, vol. 60, no. 2, pp. 529-539, 2012.

[8] X. Artiga, B. Devillers, and J. Perruisseau-Carrier, "On the selection of radiating elements for compact indoor massive-multiple input multiple output base stations," IET Microwaves, Antennas \& Propagation, vol. 8, no. 1, pp. 1-9, 2014.

[9] R. Janaswamy, "Effect of element mutual coupling on the capacity of fixed length linear arrays," IEEE Antennas and Wireless Propagation Letters, vol. 1, pp. 157-160, 2002.

[10] J. W. Wallace and M. A. Jensen, "Mutual coupling in MIMO wireless systems: a rigorous network theory analysis," IEEE Transactions on Wireless Communications, vol. 3, no. 4, pp. 13171325, 2004.

[11] M. J. Gans, "Channel capacity between antenna arrays-Part II: amplifier noise dominates," IEEE Transactions on Communications, vol. 54, no. 11, pp. 1983-1992, 2006.

[12] M. L. Morris, M. A. Jensen, and J. W. Wallace, "Superdirectivity in MIMO systems," IEEE Transactions on Antennas and Propagation, vol. 53, no. 9, pp. 2850-2857, 2005.

[13] N. W. Bikhazi and M. A. Jensen, "The relationship between antenna loss and superdirectivity in MIMO systems," IEEE Transactions on Wireless Communications, vol. 6, no. 5, pp. 1796-1802, 2007. 
[14] C. Domizioli, B. Hughes, K. Gard, and G. Lazzi, "Noise correlation in compact diversity receivers," IEEE Transactions on Communications, vol. 58, no. 5, pp. 1426-1436, 2010.

[15] C. P. Domizioli and B. L. Hughes, "Front-end design for compact MIMO receivers: a communication theory perspective," IEEE Transactions on Communications, vol. 60, no. 10, pp. 29382949, 2012.

[16] M. J. Gans, "Channel capacity between antenna arrays. Part I. Sky noise dominates," IEEE Transactions on Communications, vol. 54, no. 9, pp. 1586-1592, 2006.

[17] H. L. VanTrees, Detection Estimation and Modulation Theory: Part IV, John Wiley \& Sons, New York, NY, USA, 2002.

[18] D. Nie, B. M. Hochwald, and E. Stauffer, "Systematic design of large-scale multiport decoupling networks," IEEE Transactions on Circuits and Systems I: Regular Papers, vol. 61, no. 7, pp. 21722181, 2014.

[19] R. Mohammadkhani and J. S. Thompson, "Adaptive uncoupled termination for coupled arrays in MIMO systems," IEEE Transactions on Antennas and Propagation, vol. 61, no. 8, pp. 42844295, 2013.

[20] M. T. Ivrlac and J. A. Nossek, "Toward a circuit theory of communication," IEEE Transactions on Circuits and Systems. I. Regular Papers, vol. 57, no. 7, pp. 1663-1683, 2010.

[21] H. A. Haus and R. B. Adler, Circuit Theory of Linear Noisy Networks, The MIT Press, 1959.

[22] H. Rothe and W. Dahlke, “Theory of noisy fourpoles," Proceedings of the IRE, vol. 44, no. 6, pp. 811-818, 1956.

[23] S. Kay, Fundamentals of Statistical Signal Processing: Estimation Theory, vol. 1, Prentice Hall, Upper Saddle River, NJ, USA, 1993.

[24] J. G. Proakis, Digital Communications, McGraw-Hill, New York, NY, USA, 2001.

[25] C. A. Balanis, Antenna Theory, John Wiley \& Sons, New York, NY, USA, 1997.

[26] R. Vaughan, Channels, Propagation and Antennas for Mobile Communications, Iet, 2003.

[27] J. D. Kraus and R. J. Marhefka, Antennas For All Applications, McGraw-Hill, 2002.

[28] Y. T. Lo, S. W. Lee, and Q. H. Lee, "Optimization of directivity and signal-to-noise ratio of an arbitrary antenna array," Proceedings of the IEEE, vol. 54, no. 8, pp. 1033-1045, 1966.

[29] L. Sun, P. Li, M. R. McKay, and R. Murch, "Capacity of MIMO systems with mutual coupling: transmitter optimization with dual power constraints," IEEE Transactions on Signal Processing, vol. 60, no. 2, pp. 848-861, 2012. 

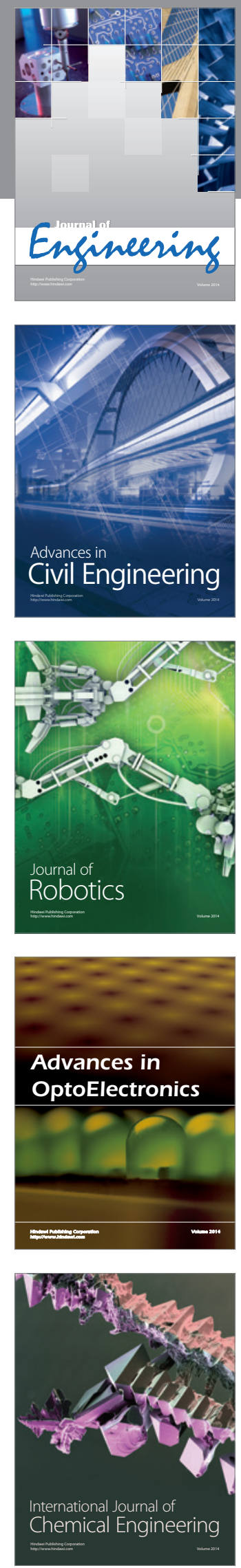

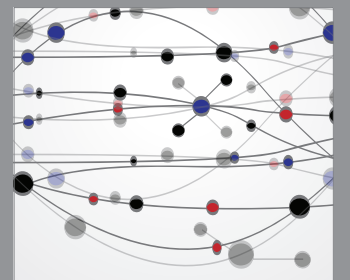

The Scientific World Journal
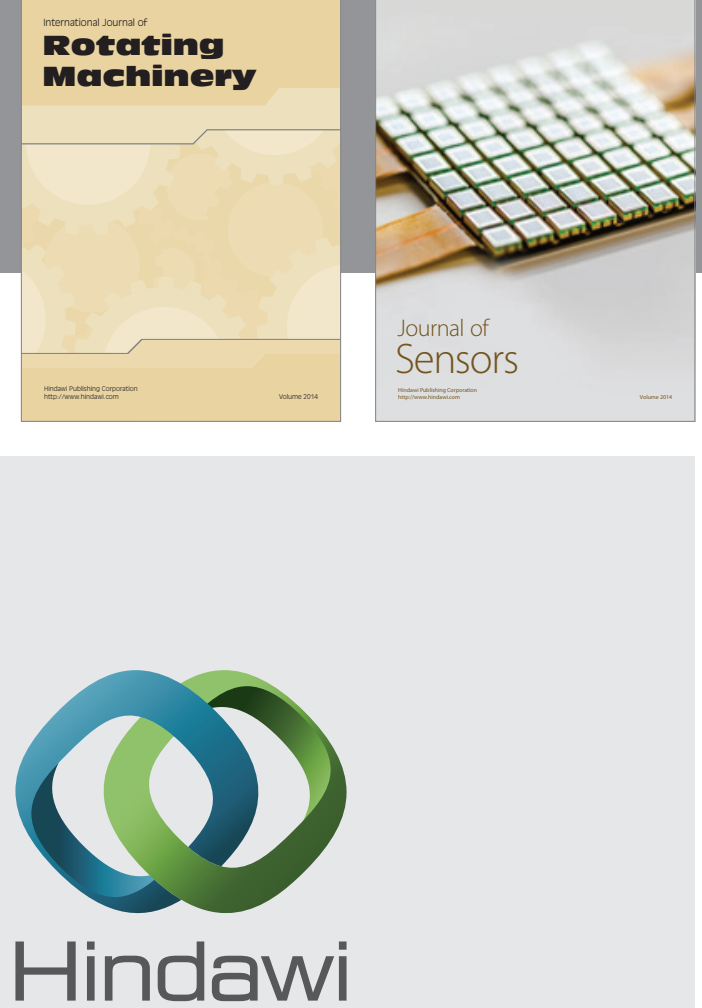

Submit your manuscripts at http://www.hindawi.com
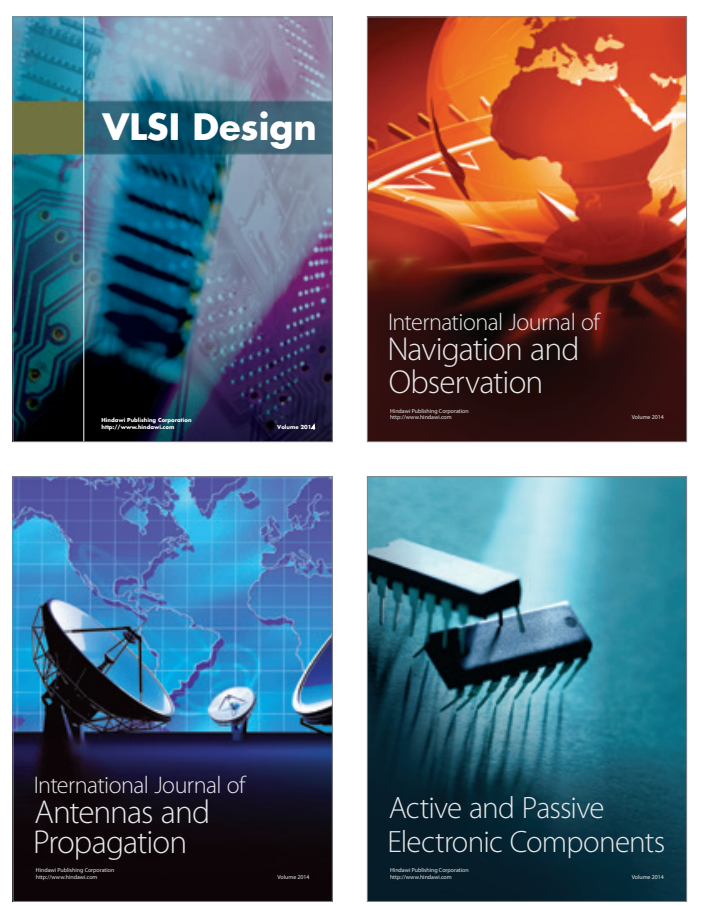
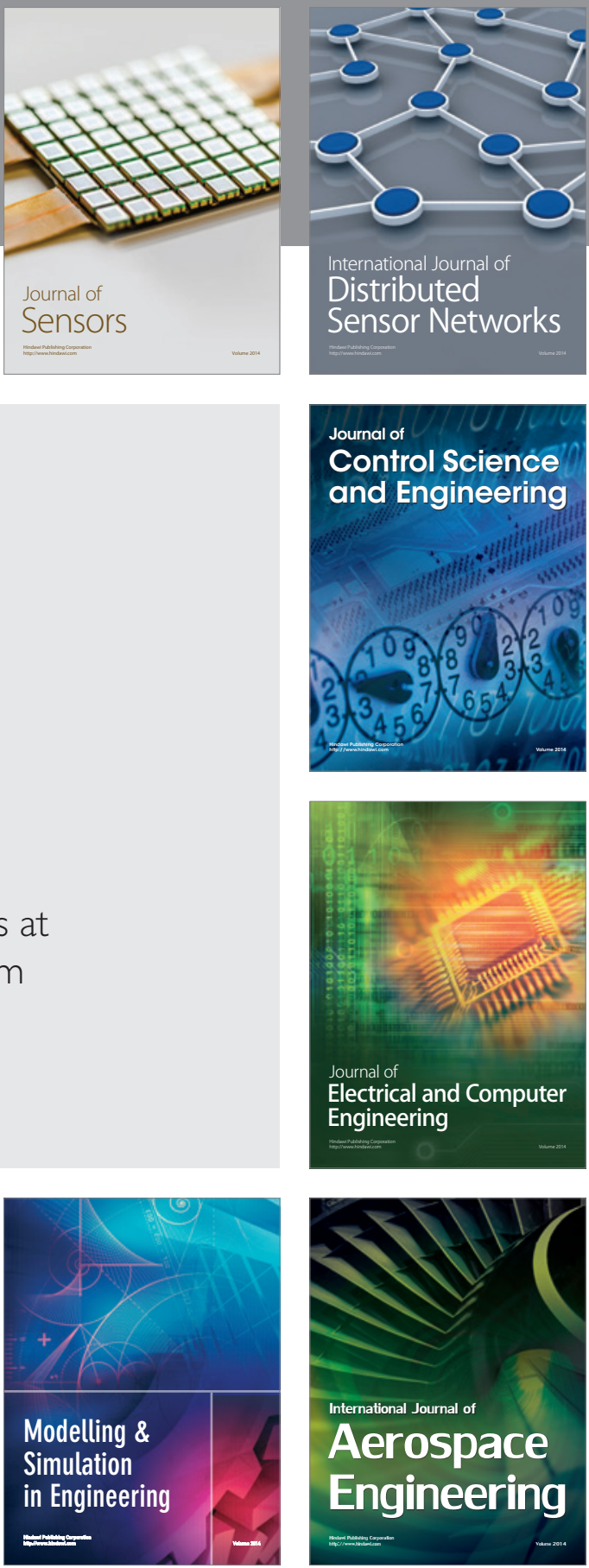

Journal of

Control Science

and Engineering
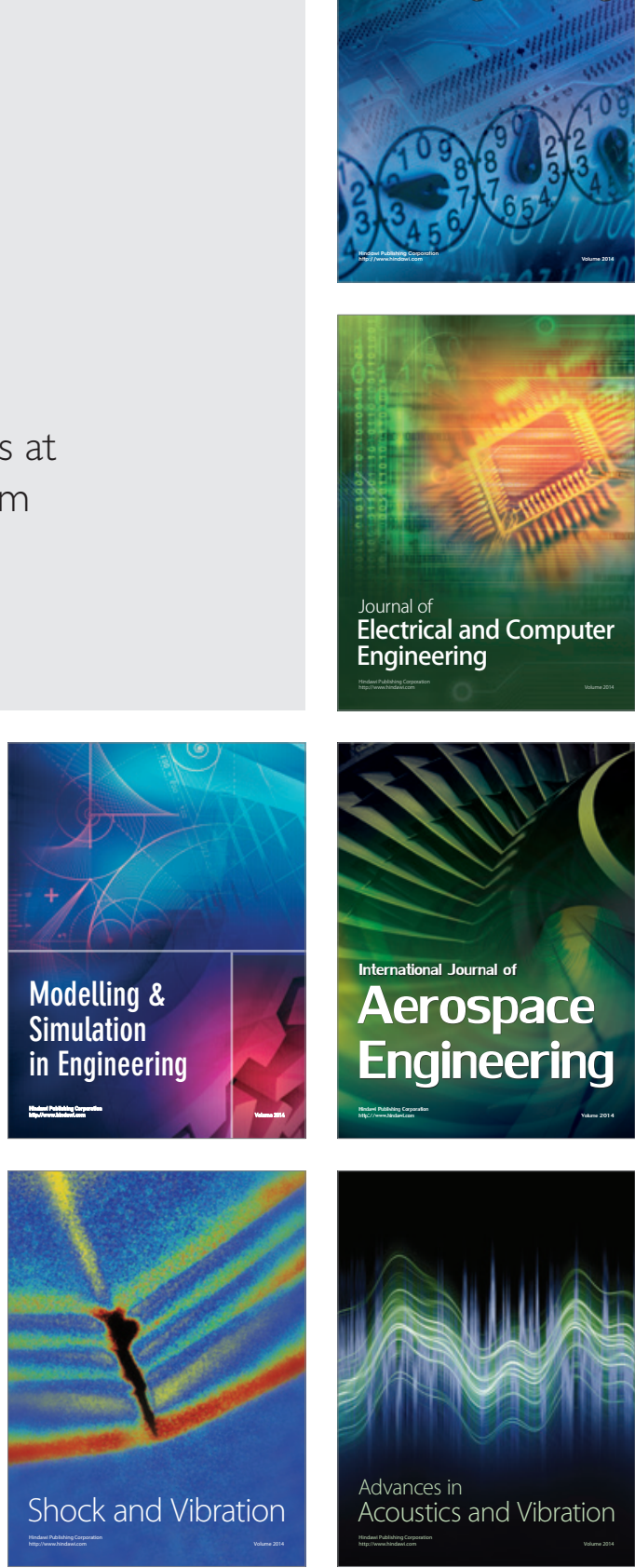